This item was submitted to Loughborough's Research Repository by the author.

Items in Figshare are protected by copyright, with all rights reserved, unless otherwise indicated.

\title{
Simultaneously enhancing mechanical properties and electrical conductivity of aluminum by using graphene as the reinforcement
}

\section{PLEASE CITE THE PUBLISHED VERSION}

https://doi.org/10.1016/j.matlet.2020.127440

PUBLISHER

ELSEVIER

VERSION

AM (Accepted Manuscript)

\section{PUBLISHER STATEMENT}

This paper was accepted for publication in the journal Materials Letters and the definitive published version is available at https://doi.org/10.1016/j.matlet.2020.127440

\section{LICENCE}

CC BY-NC-ND 4.0

\section{REPOSITORY RECORD}

Zhang, Shuai, Gaoqiang Chen, Timing Qu, Gang Fang, Shengwen Bai, Yufan Yan, Gong Zhang, et al.. 2020. "Simultaneously Enhancing Mechanical Properties and Electrical Conductivity of Aluminum by Using Graphene as the Reinforcement". Loughborough University. https://hdl.handle.net/2134/13636709.v1. 


\title{
Simultaneously enhancing mechanical properties and electrical conductivity of aluminum by using graphene as the reinforcement
}

Shuai Zhang a , Gaoqiang Chen a, Timing Qu a , Gang Fang ${ }^{\text {a }}$, Shengwen Bai ${ }^{\text {a }}$, Yufan Yan ${ }^{\text {a }}$, Gong Zhang ${ }^{\text {a }}$, Zhaoxia Zhou ${ }^{\text {b }}$, Junjun Shen ${ }^{\text {c }}$, Dawei Yao ${ }^{\text {d, }}$ Yuanwang Zhang ${ }^{\text {d }}$, Qingyu Shi ${ }^{\text {a** }}$

${ }^{a}$ State Key Laboratory of Tribology, Key Laboratory for Advanced Materials Processing Technology Ministry of Education of China, Department of Mechanical Engineering, Tsinghua University, Beijing

${ }^{\mathrm{b}}$ Loughborough materials characterization center, Department of materials, Loughborough University, Loughborough, LE11 3TU UK

${ }^{c}$ Department of Solid-State Joining Processes, Materials Mechanics, Institute of Materials Research, Helmholtz-Zentrum Geesthacht GmbH, Geesthacht, 21502, Germany

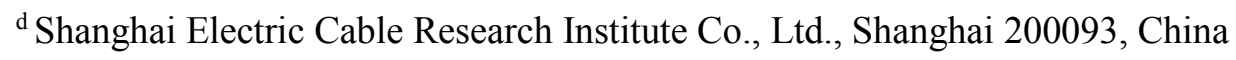

\begin{abstract}
In this study, we demonstrated simultaneously enhanced mechanical properties and electrical conductivity of pure aluminum (Al) by incorporating graphene as the reinforcement. The graphene/Al nanocomposite was fabricated by friction stir processing (FSP) combined with hot extrusion. It was found that graphene was homogeneously dispersed into $\mathrm{Al}$ matrix and directly bonded graphene/Al interfaces were formed, which were the structure basis for the simultaneously improved electrical and mechanical properties. This study provides new strategy for fabricating high-strength and highly conductive graphene/Al nanocomposites.

*Corresponding author. E-mail address: shqy@mail.tsinghua.edu.cn
\end{abstract}


Keywords: Metallic composites; Nanoparticles; Mechanical properties; Electrical properties; Friction stir processing

\section{Introduction}

Simultaneously enhancing the mechanical properties and electrical conductivity of highly conductive metals such as silver $(\mathrm{Ag})$, copper $(\mathrm{Cu})$ and aluminum $(\mathrm{Al})$ is extremely attractive for their widespread applications in electrical and electronics engineering [1], though highly challenging. Electrical conductivity is sensitive to the microstructure of conducting metallic materials, mechanically strengthening metals via alloying, precipitation and/or introducing defects such as grain boundaries and dislocations usually increases the scattering of electrons and thus decreases the electrical conductivity. Carbon nanotubes (CNTs) [2] have been attracted much interest due to their superior mechanical and physical properties such as ultra-high strength and electrical conductivity, which are expected to be potential reinforcements for enhancing the mechanical and electrical properties of conducting metallic materials [3].

However, previous studies showed that the mechanical strength of pure metals could be improved by using CNTs as reinforcements, but the electrical conductivity of these nanocomposites were always lower than that of pure metals [4-11]. The improved mechanical strength was due to the hindering of dislocation motion by CNTs $[10,11]$, while the large-sized CNTs clusters and poor interfacial bonding such as voids, cracks or impurities existed at the interfaces were the main factors causing the decrease in electrical conductivity $[4,8,10,11]$.

Ebbesen et al [2] and Wang et al [12] measured the electrical conductivity of CNTs *Corresponding author. E-mail address: shqy@mail.tsinghua.edu.cn 
and graphene, respectively. The results showed that the electrical conductivity of graphene $(\backsim 67 \mathrm{MS} / \mathrm{m})$ was significantly higher than that of CNTs $(\backsim 20 \mathrm{MS} / \mathrm{m})$. At the same time, studies focused on the electrical properties of graphene reinforced $\mathrm{Al}$ nanocomposite were limited $[13,14]$. Thus, in this study, we chose graphene as the reinforcement to enhance the mechanical properties and electrical conductivity of pure Al.

\section{Experimental procedures}

Commercial pure 1060Al plates were used as the metal matrix. Fig. 1 shows the schematic diagram of the fabrication process. Three Al plates were employed and their dimensions were $300 \times 100 \times 3 \mathrm{~mm}$ (length $\times$ width $\times$ thickness). Five slots (size: $180 \times 3 \times 3 \mathrm{~mm}$, length $\times$ width $\times$ depth) with a $3 \mathrm{~mm}$ interval were machined on middle plate and were used to fill with graphene $(0.2 \mathrm{~g}$ total weight). The three plates were then assembled to seal graphene in the middle plate. A rotating (750rpm) nonconsume tool gradually plunged into Al plates and transferred $(60 \mathrm{~mm} / \mathrm{min})$ through the region containing the slots. Partially-overlapped three-pass FSP with an offset of $3 \mathrm{~mm}$ between the adjacent passes was carried out. The graphene was distributed in the processed zone. Some graphene might escape from the gap between the plates, but the escaped graphene should be less than $10 \%$ of the added graphene. The dimensions of the processed zone were $(186 \pm 3) \times(33 \pm 3) \times(5.2 \pm 0.5) \mathrm{mm}$. Thus, the estimated weight percent of graphene was about $0.17 \% \backsim 0.29 \%$. After FSP, the graphene/Al nanocomposites were subjected to hot extrusion at temperature of $350^{\circ} \mathrm{C}$. The extrusion ratio and ram speed were 16.5:1 and $2 \mathrm{~mm} / \mathrm{s}$. Finally, hot-extruded nanocomposite sheet 
(3mm in thickness, $30 \mathrm{~mm}$ in width) was obtained.

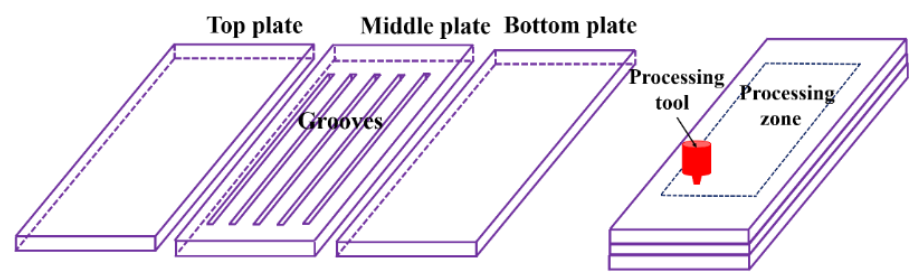

Fig.1 Schematic diagram of the FSP process.

The electrical conductivity was measured by four-probe based method at room temperature. Three nanocomposite samples with the dimensions of $50 \times 5 \times 3 \mathrm{~mm}$ (length $\times$ width $\times$ thickness) were used for conductivity measurement. Three samples with a gauge length of $3.0 \mathrm{~mm}$, a width of $2.0 \mathrm{~mm}$ and a thickness of $1.0 \mathrm{~mm}$ were used for tensile tests, which were carried out at a strain rate of $1.0 \times 10^{-3} s^{-1}$ at room temperature. For comparison, the electrical and mechanical properties testing of pure Al were carried out using the same parameters. Composite microstructures were investigated using Raman spectroscopy (wavelength:532nm), field emission scanning electron microscope (FE-SEM, Quanta 200FEG) and transmission electron microscope (TEM, JEOL JEM-2100F). Samples used for SEM analysis were pre-polished by using diamond paste $(0.5 \mu \mathrm{m})$ and fine polished by electrolytic polishing. Conventional Argon ion milling was used to fabricate the samples used for TEM analysis.

\section{Results and discussion}

Fig.2 showed the electrical properties and tensile stress-strain curves of graphene/Al nanocomposite and pure $\mathrm{Al}$, and the detailed information was shown in table.1. The measured I-V curves were changed into the form shown in Fig.2 (a) based on the equations:

*Corresponding author. E-mail address: shqy@mail.tsinghua.edu.cn 


$$
\mathrm{R}=\rho \times \frac{\mathrm{L}}{\mathrm{S}}=\frac{1}{\sigma} \times \frac{\mathrm{L}}{\mathrm{S}}=\frac{\mathrm{U}}{\mathrm{I}} \quad(1), \quad \sigma=\frac{\mathrm{I} \times \mathrm{L}}{\mathrm{U} \times \mathrm{S}}
$$

Where I was the current, $\mathrm{U}$ was the voltage, $\mathrm{L}$ was the measured length, $\mathrm{S}$ was the area of the cross-section of samples, $\sigma$ was the electrical conductivity. It was found that with the addition of graphene, the tensile strength and elongation of Al were significantly improved by $17.3 \%$ and $35.4 \%$. Meanwhile, the electrical conductivity of graphene/Al nanocomposite was $2.1 \%$ higher than that of pure Al. Although the enhancement in electrical conductivity was small, at least, the results shown in this study indicated that enhancing the mechanical properties of pure Al without sacrificing its electrical conductivity was possible. Fig.2 (c) showed the comparison results of the change in electrical conductivity and tensile strength of pure metals due to incorporation of graphene (or CNTs) into pure metal matrix between previous studies $[4-11,13,14]$ and this study.
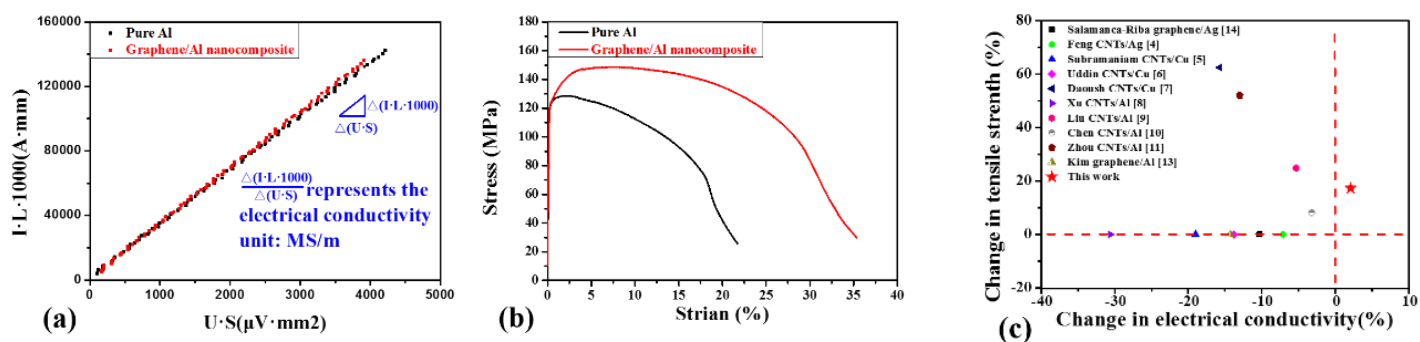

Fig.2 (a) Electrical property. (b) Tensile properties of graphene/Al nanocomposite and pure Al. (c) Comparison of the change in electrical conductivity and tensile strength of pure metals between previous studies and this study (Tensile strength of composites were not reported in references 4-6, $8,13,14)$

Table.1 Electrical conductivity and mechanical properties of graphene/Al nanocomposite and Al.

Sample Electrical conductivity (MS/m) Tensile strength (MPa) Elongation (\%) 


\begin{tabular}{cccc}
\hline Pure Al & $33.42 \pm 0.05$ & $127.16 \pm 2.50$ & $21.65 \pm 1.65$ \\
\hline Graphene/Al & $34.12 \pm 0.05$ & $149.15 \pm 12.89$ & $29.32 \pm 5.76$ \\
\hline
\end{tabular}

Five locations were randomly selected on the cross section of graphene/Al nanocomposite sheet for Raman analysis, as shown in Fig.3 (a) (Fig.3 (a) was part of the cross section of graphene/Al nanocomposite sheet). It could be clearly seen from Fig.3 (b) that graphene was detected at all the locations, which indicated that the graphene was homogeneously dispersed into Al matrix. In addition, no graphene agglomeration was found from the nanocomposite at higher magnification in SEM (Fig.3 (c)), which also showed that graphene was homogeneously dispersed into Al matrix by FSP [15]. Fig.3 (e) showed the graphene/Al interfacial bonding characteristics. No cracks, voids or aluminum carbide $\left(\mathrm{Al}_{3} \mathrm{C}_{4}\right)$ were found between graphene and $\mathrm{Al}$, which indicated that clean and intimate contact interfaces were formed under the thermomechanical conditions during the fabrication process.
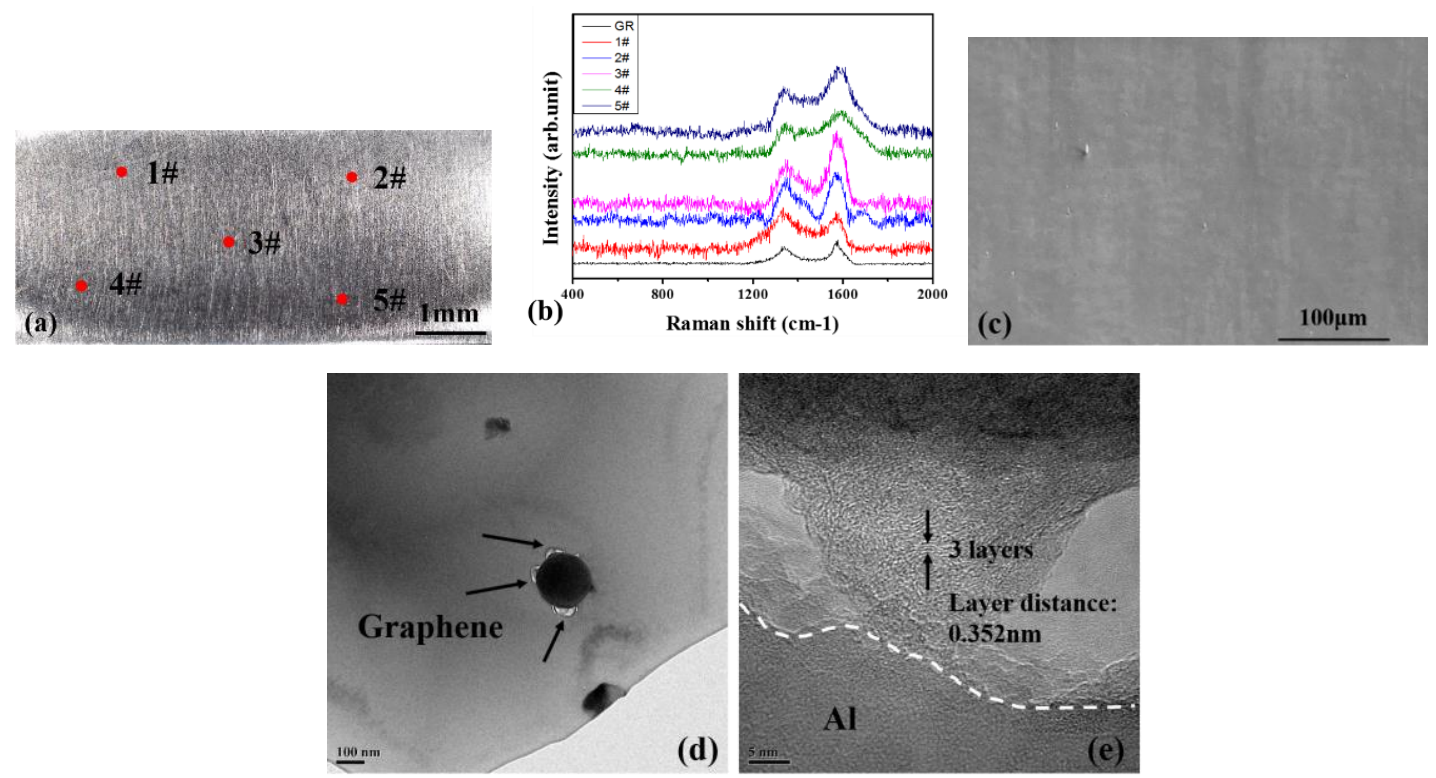

Fig.3 (a, b) Raman analysis of graphene distribution in nanocomposite. (c, d) Observation of graphene distribution in the nanocomposite by SEM and TEM. (e) Interfacial bonding *Corresponding author. E-mail address: shqy@mail.tsinghua.edu.cn 
characteristics between graphene and $\mathrm{Al}$.

The observed breakthrough of trade-off tendency between mechanical strength and electrical conductivity in this study are linked to the graphene-induced microstructures. During plastic deformation of the composite, the embedded graphene will hinder the dislocation motion within $\mathrm{Al}$ matrix and the load can be transferred from Al matrix to graphene through the interfaces [16], thus the mechanical strength of the nanocomposite is improved.

However, due to the incorporation of graphene, two competing opposite factors, which affect the electrical conductivity of graphene/Al nanocomposite, may arise. On the one hand, the excellent intrinsic electrical conductivity of graphene [12] is a positive factor for enhancing the electrical conductivity of Al matrix. Moreover, the electrical conductivity of graphene embedded in Al matrix can be further improved due to the doping effect [17]. On the other hand, the extra interfaces between graphene and $\mathrm{Al}$ is a negative factor for enhancing the electrical conductivity of the nanocomposite. Sutton et al [18] and Dong et al [19] concluded that different types of interfaces had large variations in electrical resistivity and the adverse effect of interfaces on the electrical conductivity of system can be significantly weakened by forming clean and intimate contact interfaces. It is implied that the structure manner in our graphene/Al nanocomposite, including the graphene distribution and the interfacial bonding, is the main reason for the fact that both the electrical conductivity and the mechanical strength of the graphene/Al nanocomposite fabricated in this study are higher than that of pure Al.

*Corresponding author. E-mail address: shqy@mail.tsinghua.edu.cn 


\section{Conclusions}

In summary, the simultaneously enhanced electrical conductivity and mechanical properties of Al were successfully achieved in this study by using graphene as the reinforcement. The electrical conductivity, tensile strength, and elongation of graphene/Al nanocomposite were $2.1 \%, 17.3 \%$ and $35.4 \%$ higher than those of pure Al. The observed breakthrough of trade-off tendency between mechanical properties and electrical conductivity was due to the homogeneously dispersed graphene in nanocomposite and formation of high-quality graphene/Al interfaces. This study provide new strategy for obtaining high-strength and highly-conductive graphene/Al nanocomposites.

\section{References}

[1]Lei Lu, Yongfeng Shen, Xianhua Chen, Lihua Qian and K. Lu, Science 304.5669 (2004): 422426.

[2]Ebbesen, T. W, H. J. Lezec, H. Hiura, J. W. Bennett, H. F. Ghaemi and T. Thio, Nature 382.6586 (1996): 54.

[3]Hjortstam, O, P. Isberg, S. S “oderholm, H. Dai, Applied Physics A 78.8 (2004): 1175-1179.

[4]Yi Feng, Hai Long Yuan and Min Zhang, Materials characterization 55.3 (2005): 211-218.

[5]Chandramouli Subramaniam, Takeo Yamada, Kazufumi Kobashi, Atsuko Sekiguchi, Don N. Futaba, Motoo Yumura and Kenji Hata, Nature communications 4 (2013): 2202.

[6]Sheikh M. Uddin, Tanvir Mahmud, Christoph Wolf, Carsten Glanz, Ivica Kolaric, Christoph Volkmer, Helmut Höller, Ulrich Wienecke, Siegmar Roth and Hans-Jörg Fecht, Composites Science and Technology 70 (2010): 2253-2257.

*Corresponding author. E-mail address: shqy@mail.tsinghua.edu.cn 
[7]Walid M. Daoush, Byung K. Lim, Chan B. Mo, Dong H. Nam and Soon H. Hong, Materials Science and Engineering: A 513 (2009): 247-253.

[8]C.L. Xu, B.Q. Wei , R.Z. Ma, J. Liang, X.K. Ma and D.H. Wu, Carbon 37.5 (1999): 855-858.

[9]Z.Y. Liu, B.L. Xiao, W.G. Wang and Z.Y. Ma, Journal of Materials Science \& Technology 30.7 (2014): 649-655.

[10]Biao Chen, Shufeng Li, Hisashi Imai, Lei Jia, Junko Umeda, Makoto Takahashi, and Katsuyoshi Kondoh, Journal of Alloys and Compounds 651 (2015): 608-615.

[11]Zhou Weiwei, Go Yamamoto, Yuchi Fan, Hansang Kwon, Toshiyuki Hashida, Akira Kawasaki, Carbon 106 (2016): 37-47.

[12]L. Wang, I. Meric, P. Y. Huang, Q. Gao, Y. Gao, H. Tran, T. Taniguchi, K. Watanabe, L. M. Campos, D. A. Muller, J. Guo, P. Kim, J. Hone, K. L. Shepard and C. R. Dean, Science 342.6158 (2013): 614-617.

[13]Daeyoung Kim, Seungjin Nam, Aeran Roh, Seonghyeon Yoo, Manuel Quevedo-Lopez and Hyunjoo Choi, Journal of Materials Science 52.20 (2017): 12001-12012.

[14]Lourdes G. Salamanca-Riba, Romaine A. Isaacs, Melburne C. LeMieux , Jiayu Wan , Karen Gaskell, Yeping Jiang, Manfred Wuttig, Azzam N. Mansour, Sergey N. Rashkeev, Maija M. Kuklja , Peter Y. Zavalij , Jaime R. Santiago , and Liangbing Hu, Advanced Functional Materials 25.30 (2015): 4768-4777.

[15]Hossein Izadi, Adrian P. Gerlich, Carbon 50 (2012):4744 -4749.

[16]Z.W. Zhang, Z.Y. Liu, B.L. Xiao, D.R. Ni, Z.Y. Ma, Carbon 135 (2018): 215-223.

[17]Mu Cao, Yongzhi Luo, Yiqun Xie, Zhanqiu Tan, Genlian Fan, Qiang Guo, Yishi Su, Zhiqiang Li, and Ding-Bang Xiong, Advanced Materials Interfaces (2019): 1900468.

*Corresponding author. E-mail address: shqy@mail.tsinghua.edu.cn 
[18]A. P. Sutton, R. W. Balluffi, Interfaces in Crystalline Materials (Clarendon, Oxford, 1995).

[19]Lifeng Dong, Steven Youkey, Jocelyn Bush, and Jun Jiao, Journal of applied physics 101.2 (2007): 024320.

*Corresponding author. E-mail address: shqy@mail.tsinghua.edu.cn 